\title{
Clinical characteristics and outcome of patients with status myoclonus following out of hospital cardiac arrest
}

\author{
MI Ruknuddeen ${ }^{1 *}$, V Rajajee ${ }^{2}$, R Ramadoss ${ }^{3}$, L Grzeskowiak ${ }^{4}$, R Rajagopalan ${ }^{5}$ \\ From ESICM LIVES 2015 \\ Berlin, Germany. 3-7 October 2015
}

\section{Introduction}

Generalized status myoclonus following out of hospital cardiac arrest (OHCA) has been associated with poor prognosis ${ }^{1}$. Many patients die and majority of those who survive remain in a vegetative state ${ }^{2}$. There is paucity of data on the clinical characteristics and outcome of patients with status myoclonus treated with hypothermia from resource-limited centres in South Asia.

\section{Objectives}

To study the clinical characteristics and outcome of patients with status myoclonus following out of hospital cardiac arrest and treated with hypothermia.

\section{Methods}

Retrospective chart review of patients surviving $>24$ hours following OHCA in an urban community hospital in South India, between January 2006 and December 2012. All patients received therapeutic hypothermia $\left(32-34^{\circ} \mathrm{C}\right)$ for 24 hours. Data on the clinical characteristics of patients developing status myoclonus (clinical myoclonic status epilepticus) and their outcome (survival, morbidity and mortality) were studied in detail. Based on the cerebral performance category (CPC) score at hospital discharge, the neurological outcome of patients was classified into favorable $(\mathrm{CPC} 1,2)$ and unfavorable (CPC 3,4).

\section{Results}

Out of the 124 patients surviving $>24$ hours, 52 (42\%) developed status myoclonus. Baseline and general characteristics were similar in patients with and without status myoclonus (Table 1). Majority of patients with status myoclonus had absent brainstem reflexes [(pupillary, corneal and cough) $92 \%$ vs. $62 \%, \mathrm{P}<0.001]$ and motor response worse than flexion $(100 \%$ vs. $71 \%, \mathrm{P}<0.001)$ on day 3 post-cardiac arrest (Table 2). None of the patients with status myoclonus had favorable neurological outcome (50 patients died and 2 patients were discharged in vegetative

Table 1. General Characteristics

\begin{tabular}{llll}
\hline Characteristics & Status Myoclonus Present N= 52 & Status Myoclonus Absent N= 72 & P Value \\
\hline Duration of cardiac arrest >20 minutes N (\%) & $50(96.2)$ & $60(87.0)$ & 0.113 \\
\hline Charlson comorbidity index Median (IQR) & $3(2-6)$ & $5(2-6)$ & 0.328 \\
\hline APACHE II score Median (IQR) & $34(30-37.5)$ & $35(31-40)$ & 0.420 \\
\hline Sex N (\%) Male Female & $33(63.5) 19(36.5)$ & $44(63.8) 25(36.2)$ & 0.972 \\
\hline Age Median Years (IQR) & $60(47.5-67.5)$ & $63(54-70)$ & 0.250 \\
\hline Metabolic Acidemia at presentation N (\%) PH <7.0 & $49(94.2)$ & $63(91.3)$ & 0.544 \\
\hline Etiology of cardiac arrest: Non-cardiac N (\%) & $36(69.2)$ & $37(53.6)$ & 0.082 \\
\hline Non-shockable rhythm N (\%) & $50(96.2)$ & $59(85.5)$ & 0.052 \\
\hline
\end{tabular}

'Lyell McEwin Hospital, Intensive Care Medicine, Adelaide, Australia

Full list of author information is available at the end of the article 
Table 2. Neurology, Morbidity and Mortality

\begin{tabular}{|c|c|c|c|}
\hline Neurological Characteristics & $\begin{array}{l}\text { Status Myoclonus Present } \mathrm{N}= \\
52\end{array}$ & $\begin{array}{l}\text { Status Myoclonus Absent } \mathrm{N}= \\
72\end{array}$ & $P$ value \\
\hline Absent brainstem reflexes on day 3, post-cardiac arrest $\mathrm{N}(\%)$ & $47(90.4)$ & $43(62.3 \%)$ & $<+001$ \\
\hline Motor response worse than flexion, day 3 post-cardiac arrest $\mathrm{N}(\%)$ & $52(100)$ & $49(71)$ & $\begin{array}{c}< \\
0.001\end{array}$ \\
\hline$\overline{C P C} 1 \mathrm{~N}(\%)$ & 0 & $18(25 \%)$ & $\begin{array}{c}< \\
0.001\end{array}$ \\
\hline CPC 4 (N \%) & $2(4)$ & & \\
\hline Mortality N (\%) 104 (84) & $50(96)$ & $54(75)$ & $<+001$ \\
\hline Death Due to underlying illness N (\%) 51 (49) & $13(26)$ & $38(70.4)$ & $\begin{array}{c}< \\
0.001\end{array}$ \\
\hline $\begin{array}{l}\text { Death following withdrawal of life sustaining treatment N (\%) } \\
53 \text { (51) }\end{array}$ & $37(74)$ & $16(29.6)$ & $\begin{array}{c}< \\
0.001\end{array}$ \\
\hline
\end{tabular}

state, CPC 4). Out of the 72 patients without status myoclonus, 18 (25\%) patients had favorable neurological outcome (CPC1). Out of the 104 patients who died, 53 (51\%) were following withdrawal of life sustaining treatment (WLST) and majority $(37 / 53)$ of these patients had status myoclonus $(74 \%$ vs. $30 \%, \mathrm{P}<0.001)$.

\section{Conclusions}

Status myoclonus following out of hospital cardiac arrest was associated with unfavorable outcome. Majority of deaths in patients who developed status myoclonus was secondary to WLST, which may indicate self-fulfilling prophecy.

\section{Authors' details}

${ }^{1}$ Lyell McEwin Hospital, Intensive Care Medicine, Adelaide, Australia.

${ }^{2}$ University of Michigan, Neurosurgery and Neurology, Michigan, United States. ${ }^{3}$ University of Adelaide/Lyell McEwin Hospital, Intensive Care Medicine, Adelaide, Australia. ${ }^{4}$ University of Adelaide/The Robinson Research Institute, Adelaide, Australia. ${ }^{5}$ Sundaram Medical Foundation, Intensive Care Medicine, Chennai, India.

Published: 1 October 2015

\section{References}

1. Thomke F, Markx JJ, Sauer O, et al: Observations on comatose survivors of cardiopulmonary resuscitation with generalized myoclonus. BMC Neurology 2005, 5:14, Jul 18.

2. Wijdicks EF, Parisi JE, Sharbrough FW: Prognostic value of myoclonus status in comatose survivors of cardiac arrest. Annals of neurology 1994 35(2):239-43, Feb.

doi:10.1186/2197-425X-3-S1-A198

Cite this article as: Ruknuddeen et al:: Clinical characteristics and

outcome of patients with status myoclonus following out of hospital cardiac arrest. Intensive Care Medicine Experimental 2015 3(Suppl 1):A198.

\section{Submit your manuscript to a SpringerOpen ${ }^{\circ}$ journal and benefit from:}

- Convenient online submission

- Rigorous peer review

- Immediate publication on acceptance

- Open access: articles freely available online

- High visibility within the field

- Retaining the copyright to your article

Submit your next manuscript at $\boldsymbol{s p r i n g e r o p e n . c o m ~}$ 